\title{
Magnetically Levitated Planar Actuator With Moving Magnets
}

\author{
J. W. Jansen, Member, IEEE, C. M. M. van Lierop, Member, IEEE, Elena A. Lomonova, Senior Member, IEEE, \\ and André J. A. Vandenput, Senior Member, IEEE
}

\begin{abstract}
This paper concerns the design, optimization, and commutation of a six-degree-of-freedom planar actuator with an active magnetic bearing. The planar motor has a stationary coil array and a translator with a Halbach magnet array. During movements in the $x y$ plane, the set of energized coils changes with the position of the translator. In this paper, a method for the electromagnetic design of this type of actuator is discussed, and several topologies are compared. The best performing topology in terms of power dissipation and force and torque ripples has been manufactured and successfully tested.
\end{abstract}

Index Terms-Linear synchronous motors, magnetic levitation, permanent-magnet motors, planar actuators.

\section{INTRODUCTION}

$\mathbf{M}$ AGNETICALLY levitated planar actuators are developed as alternatives to $x y$ drives constructed of stacked linear motors. The translator of these actuators is suspended above the stator with no support other than magnetic fields. The gravitational force is fully counteracted by the electromagnetic force. The translator of these ironless planar actuators can move over relatively large distances in the $x y$ plane only, but it has to be controlled in six degrees of freedom (6-DOF) because of the active magnetic bearing. The advantage of magnetically levitated planar actuators is that they can operate in vacuum, for example, in extreme-UV lithography equipment. Planar actuators can be constructed in two ways. The actuator has either moving coils and stationary magnets [1] or moving magnets and stationary coils [2]-[5]. The last type of planar actuator does not require a cable to the moving part.

In this paper, a 6-DOF long-stroke moving-magnet planar actuator is investigated. Fig. 1 shows an overview of such an actuator. The stator of this motor consists of 84 coils with

Paper IPCSD-07-089, presented at the 2007 IEEE International Electric Machines and Drives Conference, Antalya, Turkey, May 3-5, and approved for publication in the IEEE TRANSACTIONS ON INDUSTRY APPLICATIONS by the Electric Machines Committee of the IEEE Industry Applications Society. Manuscript submitted for review May 30, 2007 and released for publication October 29, 2007. Published July 23, 2008 (projected). This IOP-EMVT project was supported by SenterNovem, an agency of the Dutch Ministry of Economic Affairs.

J. W. Jansen and E. A. Lomonova are with the Electromechanics and Power Electronics Group, Department of Electrical Engineering, Eindhoven University of Technology, 5612AZ Eindhoven, The Netherlands (e-mail: j.w.jansen@tue.nl; e.lomonova@tue.nl).

C. M. M. van Lierop is with the Control Systems Group, Department of Electrical Engineering, Eindhoven University of Technology, 5612AZ Eindhoven, The Netherlands (e-mail: c.m.m.v.lierop@tue.nl).

A. J. A. Vandenput, deceased, was with the Electromechanics and Power Electronics Group, Department of Electrical Engineering, Eindhoven University of Technology, 5612AZ Eindhoven, The Netherlands.

Digital Object Identifier 10.1109/TIA.2008.926065

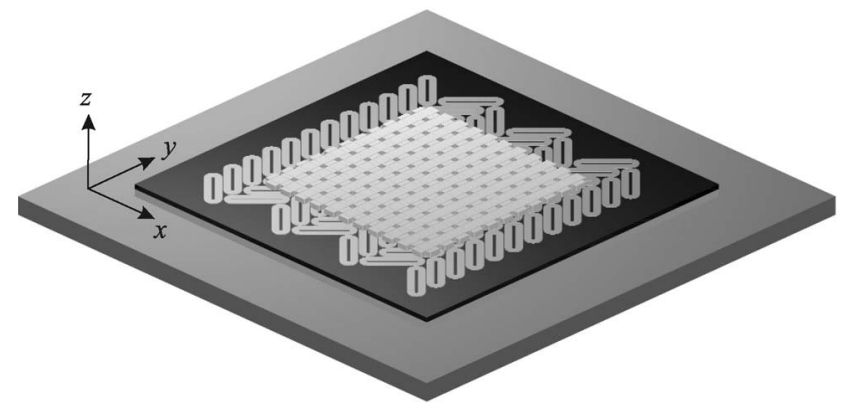

Fig. 1. Moving-magnet planar actuator.

concentrated windings of which only 24 are simultaneously energized. The moving part is a permanent-magnet array having a Halbach magnetization. During movements in the $x y$ plane, the set of active coils changes with the position of the translator because only the coils below and near the edge of the magnet array can produce significant force and torque. Although the actuator can be classified as an ac synchronous motor, DQ0 decomposition cannot be used for the decoupling of the force and the torque. Therefore, new decoupling and commutation methods have been developed, and every coil is excited with a single-phase current amplifier [6]. The current waveforms are nonsinusoidal.

In the literature, little information about the design of longstroke 6-DOF moving-magnet planar actuators can be found. This paper presents a fast design method for these actuators. Several different topologies are optimized with this method and the power dissipation, i.e., the ohmic losses, and the force and torque ripples are compared. In the last analysis, the nonlinear decoupling and switching algorithm is incorporated.

\section{Design Method}

The coils in the planar actuator are responsible for the propulsion in the $x y$ plane and the levitation of the translator. The force vector $\vec{F}$ produced by a single coil depends on the relative position of the coil with respect to the magnetic flux density distribution of the permanent magnets. The torque vector $\vec{T}$ produced by the coil depends on the distribution of the force in the volume of the coil and the distance to the mass center point of the translator. Because of the absence of iron, the force can be calculated with the Lorentz force law. In [7], three different methods for the calculation of the Lorentz force and torque in moving-magnet planar actuators are presented and verified. These models are linear with the current, and assuming 
rigid body dynamics, the total force and torque on the translator are the sum of the contributions of individual coils.

The wrench $\vec{w}$ is the vector that contains the total force and torque acting on the translator, i.e.,

$$
\vec{w}=\left[\begin{array}{llllll}
F_{x} & F_{y} & F_{z} & T_{x} & T_{y} & T_{z}
\end{array}\right]^{\top} .
$$

The wrench of a planar actuator with $n$ coils can be expressed as

$$
\begin{aligned}
\vec{w} & =\left[\begin{array}{llll}
F_{1, x}(\vec{p}) & F_{2, x}(\vec{p}) & \cdots & F_{n, x}(\vec{p}) \\
F_{1, y}(\vec{p}) & F_{2, y}(\vec{p}) & \cdots & F_{n, y}(\vec{p}) \\
F_{1, z}(\vec{p}) & F_{2, z}(\vec{p}) & \cdots & F_{n, z}(\vec{p}) \\
T_{1, x}(\vec{p}) & T_{2, x}(\vec{p}) & \cdots & T_{n, x}(\vec{p}) \\
T_{1, y}(\vec{p}) & T_{2, y}(\vec{p}) & \cdots & T_{n, y}(\vec{p}) \\
T_{1, z}(\vec{p}) & T_{2, z}(\vec{p}) & \cdots & T_{n, z}(\vec{p})
\end{array}\right]\left[\begin{array}{c}
i_{1} \\
i_{2} \\
\vdots \\
i_{n}
\end{array}\right] \\
& =\boldsymbol{\Gamma}(\vec{p}) \vec{i}
\end{aligned}
$$

where $\vec{i}$ is the current vector containing the currents in the respective coils. The matrix $\boldsymbol{\Gamma}(\vec{p})$ is a mapping of the currents in the coils on the force and torque exerted on the translator as a function of the position and orientation of the translator in 6 -DOF $\vec{p}$. The force and torque functions are expressed on a per-ampere base.

In the design process of the planar actuator, we want to determine both the shape and the distribution of the coils. Because the planar actuator is applied in accurate positioning systems, both the power dissipation and the ripples of the force and torque are important design criteria. As the simulation of planar actuators is computationally demanding, the design is split into two steps. First, a single coil and a magnet array are optimized to find efficient coil and magnet geometries. Second, with these optimized coils, planar actuators are synthesized and compared.

\section{Optimization OF A MAgnet ARRAY AND A SINGLE COIL}

The planar actuator has a single-sided active magnetic bearing. This magnetic bearing is not prestressed by permanent magnets, and consequently, a significant amount of energy is dissipated in the coils for the levitation of the translator. Therefore, reduction of the power dissipation is the main design criterion.

To reduce the power dissipation, the planar actuator should be constructed of coils that can efficiently produce force. As stated above, the coils in the planar actuator can be treated as separate elements because the superposition principle can be applied to the force and torque calculation. Therefore, a planar actuator with only a single coil is optimized to find optimal coil and magnet geometries.

There are two basic shapes for the concentrated windings in the planar actuator. Fig. 2 shows a Halbach permanent-magnet array and the two coil types. Round or square coils (coil A), which are aligned with the permanent-magnet array, can be applied [3]. The size of these coils is limited by the pole pitch $\tau$. This coil produces force in the $x-, y$-, and $z$-directions. In [1], it is proposed to rotate the coils $45^{\circ}$ with respect to the magnets

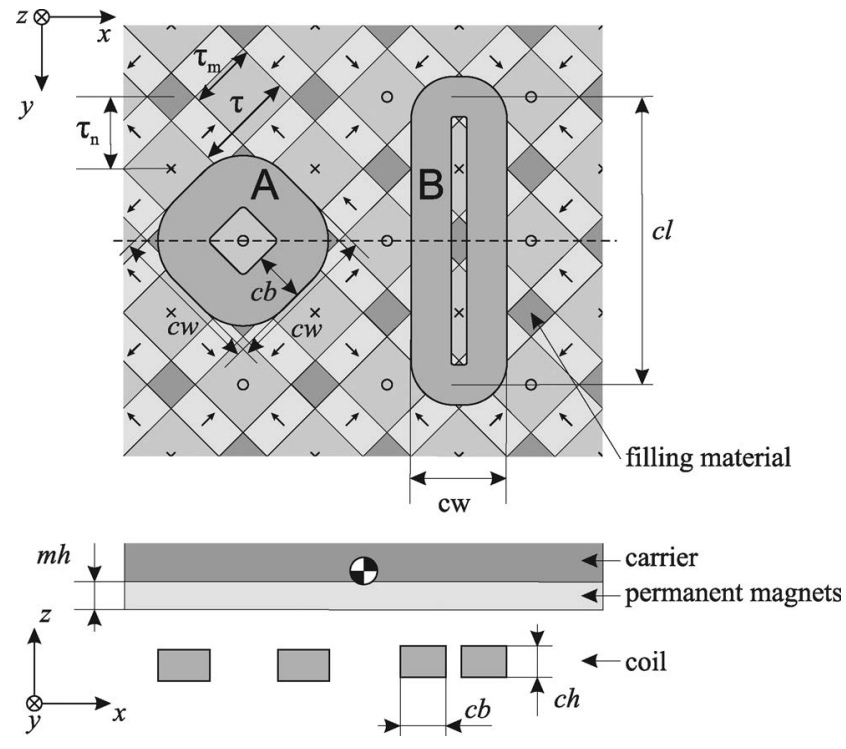

Fig. 2. Two types of concentrated coils. (a) Square/round. (b) Rectangular.

(coil B). A new pole pitch $\tau_{n}$ should be introduced to describe the rotated magnetic flux density distribution, i.e.,

$$
\tau_{n}=\frac{\tau}{\sqrt{2}} .
$$

The size of this coil is not limited to the pole pitch in the $y$-direction, as it can further be extended in the $y$-direction. If the coil length

$$
c l=2 n \tau_{n}
$$

where $n$ is an integer, ideally the coil will only produce force in the $x$ - and $z$-directions. In that case, the force vector is independent of the $y$ position. If the coil is rotated $90^{\circ}$ about the $z$-axis, it produces force in the $y z$ plane. Thus, the force production in the $x$ - and $y$-directions can physically be decoupled.

In the optimization, it is assumed that the translator of the planar actuator is levitated by a single coil. The power dissipated in that coil is minimized. The objective function $f$ is equal to

$$
f=\left(\frac{m g i}{F_{z}}\right)^{2} R
$$

where $F_{z}$ is the levitation force, $m$ is the translator mass, $g$ is the acceleration due to gravity, $i$ is the current, and $R$ is the resistance of the coil.

The optimization variables and parameters are indicated in Fig. 2 and are summarized in Table I. The optimal dimensions of the coil and magnet array (the optimization variables) are calculated by the optimization algorithm. Three different groups of parameters, which are fixed during the optimization, can be defined.

1) The pole pitch of the magnet array, the length of the coils, and the width of the coils are determined by the topology. This will further be discussed in the comparison of planar actuators. 
TABLE I

OPTIMIZATION VARIABLES AND PARAMETERS

\begin{tabular}{|l|c|c|}
\hline Optimization variable & Symbol/value & Unit \\
\hline Coil height & $c h$ & $\mathrm{~m}$ \\
Conductor bundle width & $c b$ & $\mathrm{~m}$ \\
Ratio of the magnet pitch and the pole pitch & $\tau_{m} / \tau$ & - \\
Magnet height & $m h$ & $\mathrm{~m}$ \\
\hline Optimization parameter & & \\
\hline Pole pitch & $\tau$ & $\mathrm{m}$ \\
Coil length & $c l$ & $\mathrm{~m}$ \\
Maximal coil width & $c w$ & $\mathrm{~m}$ \\
Mass carrier & 4 & $\mathrm{~kg}$ \\
Mass density permanent magnets & 7700 & $\mathrm{~kg} / \mathrm{m}^{3}$ \\
Mass density filling material & $\rho_{f}$ & $\mathrm{~kg} / \mathrm{m}^{3}$ \\
Remanence permanent magnets & 1.24 & $\mathrm{~T}$ \\
Relative permeability permanent magnets & 1.03 & - \\
\hline
\end{tabular}

2) The mass of the permanent magnets is a considerable part of the total mass of the translator. Therefore, the mass of the translator without the magnets (the mass of the carrier to which the magnets are glued) and the mass densities of the permanent magnets and the material used to fill the empty spaces in the magnet array (usually an epoxy resin) are important parameters in the optimization.

3) The magnet specifications, i.e., the remanence of the permanent magnets $B_{r}$ and the relative permeability $\mu_{r}$.

The optimization has been carried out with a Matlab function, which uses a sequential quadratic programming routine. The force has been calculated with the harmonic model presented in [7]. Using the harmonic model, it can be shown that the coil sizes (the coil height $c h$ and the conductor bundle width $c b$ ) are uncorrelated in the objective function when $c l \gg c b$. The ratios of the sizes of the magnets in the magnet array $\left(\tau_{m} / \tau\right)$ and the magnet height $m h$ are weakly correlated as they both affect the mass of the translator.

The optimal values for the magnet array and the coil array can also be obtained with separate optimizations. The optimal value for the ratio of the sizes of the permanent magnets and the magnet height can be found by maximizing the amplitude of the magnetic flux density of the magnet array per unit of mass of the translator. The optimal values of the coils can also be obtained by maximizing the steepness $S$ of the coil as

$$
S=\frac{F^{2}}{i^{2} R}
$$

The steepness is a measure to compare the power dissipation of electrical machines. It can be demonstrated that the optimal value of the coil height only depends on the pole pitch: $c h=$ $0.283 \tau$. For coil B shown in Fig. 2 with $c w=(4 / 3) \tau_{n}$ and $c l=$ $4 \tau_{n}$, the optimum value of $c b$ is equal to $c b=0.56 \tau_{n}$, which is similar to the value found in [1].

In Fig. 3, it is illustrated that the optimum value of $\left(\tau_{m} / \tau\right)$ depends on the properties of the material used to fill the empty spaces in the magnet array. Depending on its mass density, the optimal value varies between 0.64 and 0.70. In Fig. 3 , the power dissipation is shown when the levitation force is generated by a single coil. In practice, the actuator is levitated by more coils, and the total power dissipation will decrease in proportion. Furthermore, the power dissipation is shown for an actuator with a north-south (NS) magnet array, which only contains

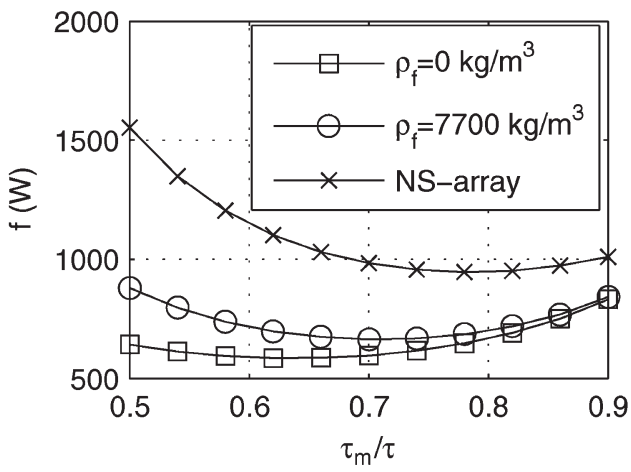

Fig. 3. Power dissipated for levitation of the translator with a single coil $\left(\tau=0.025 \mathrm{~m}, c l=4 \tau_{n}, c w=(3 / 2) \tau_{n}, B_{r}=1.24 \mathrm{~T}, \mu_{r}=1.03\right.$, and 121 $(11 \times 11)$ poles in the magnet array). For the NS array, $\rho_{f}=0 \mathrm{~kg} / \mathrm{m}^{3}$.

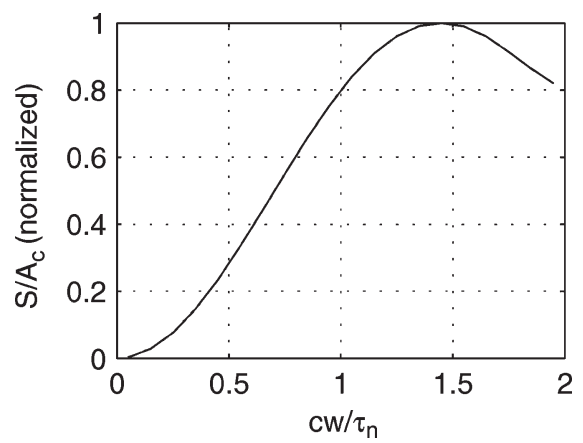

Fig. 4. Steepness per coil area versus the coil width $c w$.

magnets magnetized in the $z$-direction and no filling material in between the magnets. Fig. 3 clearly shows that a planar actuator with a Halbach permanent magnet array has a significantly lower power dissipation.

For the selection of the number of coils per pole, the force density of different coils is determined. For that purpose, the steepness $S$ per area of the coil $A_{c}$ is calculated, where $A_{c}=$ $(c l+c b) c w$ (rectangular coil). Fig. 4 shows the ratio $\left(S / A_{c}\right)$ for different values of the coil width and a fixed pole pitch. The highest values of $\left(S / A_{c}\right)$ are obtained for a coil width of $c w=(4 / 3) \tau_{n}$ (i.e., for a coil distribution according to a three-phase system) and a coil width of $c w=(3 / 2) \tau_{n}$ (i.e., for a coil distribution according to a (semi-) four-phase system). For square coils similar results are obtained. Therefore, these coil widths are used in the synthesis of the planar actuator topologies.

\section{Decoupling of the Force And the Torque}

The controller of the planar actuator calculates a force and torque reference or wrench command every sample time. Like in rotary and linear machines, this wrench command is converted to current setpoints for the power amplifiers. The current setpoints are calculated by inverting the planar actuator model $\boldsymbol{\Gamma}(\vec{p})$. Because the motor is overactuated, there is an infinitely large set of solutions for this inverse. A convenient solution is to search for the inverse with minimum power dissipation in the coils. This is similar to minimizing the 2-norm of the current 
vector $\vec{i}$. The solution is the Moore-Penrose inverse of $\boldsymbol{\Gamma}(\vec{p})$ $[8]$ as

$$
\min _{\boldsymbol{\Gamma}(\vec{p}) \vec{i}=\vec{w}}\|\vec{i}\|=\left\|\boldsymbol{\Gamma}^{-}(\vec{p}) \vec{w}\right\|
$$

where

$$
\boldsymbol{\Gamma}^{-}(\vec{p})=\boldsymbol{\Gamma}(\vec{p})^{\top}\left(\boldsymbol{\Gamma}(\vec{p}) \boldsymbol{\Gamma}(\vec{p})^{\top}\right)^{-1}
$$

when the resistances of all coils are equal. This method requires a model of the force and torque exerted by the coils, which is valid over the full stroke of the planar actuator, including all edge effects. Because only the coils beneath and near the edges of the magnet array can efficiently produce force and torque, the currents in the coils are automatically reduced near the edges of the magnet array.

For the decoupling of the planar actuators designed in this paper, a different method is applied because the available calculation time on the digital signal processor of the control hardware is limited. The force and torque exerted by the coils are modeled by a simple computation-inexpensive analytical model, which is presented in [7]. This model only takes the first harmonic of the magnetic flux density distribution into account, and the coil shapes are idealized. The end effects of the magnet array are not included in the model, and because of that, additional constraints are added to the minimization criterion. A smooth diagonal weighing matrix $\Delta$ contains these extra constraints and penalizes the coils near the edges of the magnet array. As a result, the coils will smoothly be switched off. The weighed 2-norm minimization becomes

$$
\min _{\boldsymbol{\Gamma}(\vec{p}) \vec{i}=\vec{w}}\|\vec{i}\|_{\Delta^{-1}(\vec{p})}=\left\|\boldsymbol{\Gamma}^{-}(\vec{p}) \vec{w}\right\|
$$

where

$$
\boldsymbol{\Gamma}^{-}(\vec{p})=\boldsymbol{\Delta}(\vec{p}) \boldsymbol{\Gamma}(\vec{p})^{\top}\left(\boldsymbol{\Gamma}(\vec{p}) \boldsymbol{\Delta}(\vec{p}) \boldsymbol{\Gamma}(\vec{p})^{\top}\right)^{-1} .
$$

More details about the decoupling method can be found in [6].

The effect of the weighing function is illustrated in Fig. 5. In the bottom-left part of Fig. 5, the planar actuator in Fig. 1 with 24 active coils is shown. The active coils are dark gray, and the coils that are switched off are light gray. The weighing functions do not penalize any coil. When the translator moves in the $x$-direction, the active coils in the left column will smoothly be switched off. After they are switched off, the adjacent coils in the outer right column will smoothly be switched on (not shown). At the transition, only 18 coils are active (bottom-right part of Fig. 5). When the translator moves in the $y$-direction, only 20 coils are active at the transition between two different sets of coils (top-left part of Fig. 5). In the worstcase situation (top-right part of Fig. 5), nine coils are penalized by the weighing function and are switched off.

\section{DESIGN CONSIDERATION}

Planar actuators provide a lot of design freedom because the flux paths are 3-D, the coils are individually excited, and the set of active coils changes with the position of the translator. In the design process, different factors are considered.

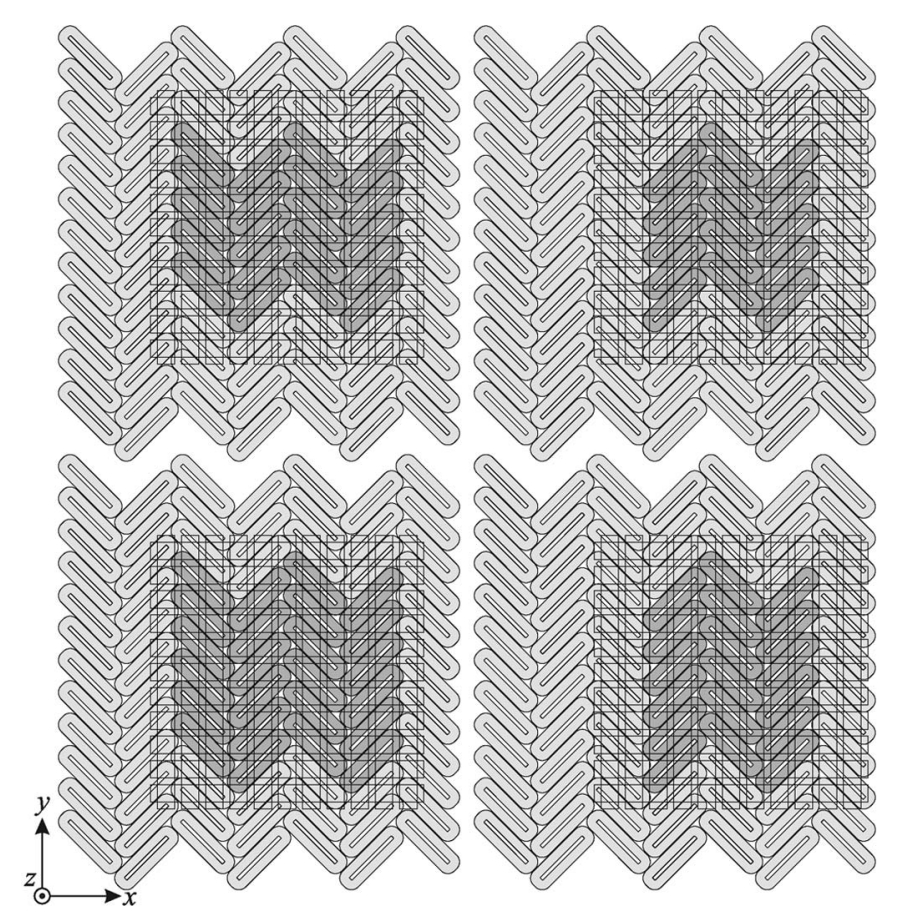

Fig. 5. Active coils at the transitions between different sets of coils.

1) Switching Between Coil Sets: As illustrated in Fig. 5, the number of active coils changes with the position of the translator. In this example, between 15 and 24 coils are active. The power dissipation in the actuator is considerably larger when a similar wrench should be produced with only 15 coils. Therefore, a large variation in the number of active coils is not desired.

2) Length of Rectangular Coils: The force on rectangular coils is mainly produced in the long straight segments of the coils. As a result, longer coils are more efficient. However, the current in the coil is penalized by the window function when the model of the coil $\boldsymbol{\Gamma}(\vec{p})$ is no longer accurate. Consequently, with the applied decoupling and switching algorithm, a longer coil should be switched off further from the edge than a shorter coil. Moreover, the physical decoupling of the force in the $x y$ plane does not hold if a rectangular coil partly leaves the surface of the magnet array. For that reason, only topologies with rectangular coils with $c l=2 \tau_{n}$ and $c l=4 \tau_{n}$ are investigated.

3) Controllability: One of the questions that arises in the design process is the controllability of a planar actuator design. The controllability can be predicted by calculating the condition number of $\boldsymbol{\Gamma}^{-}(\vec{p})$ over the stroke of the planar actuator. Because the rotation angles and the stroke in the $z$-directions are limited, calculation of the condition number on a dense grid in the $x y$ plane is in this case sufficient. The condition number of $\boldsymbol{\Gamma}^{-}(\vec{p})$ is a measure for the sensitivity of the matrix inversion to errors in the force and torque functions in the mapping. If the condition number is sufficiently small, all force and torque combinations can be accurately achieved. However, the condition number itself is not suitable for use as a design criterion as the mapping $\boldsymbol{\Gamma}(\vec{p})$ contains elements of different units (N/A and $\mathrm{N} \cdot \mathrm{m} / \mathrm{A})[9]$. 


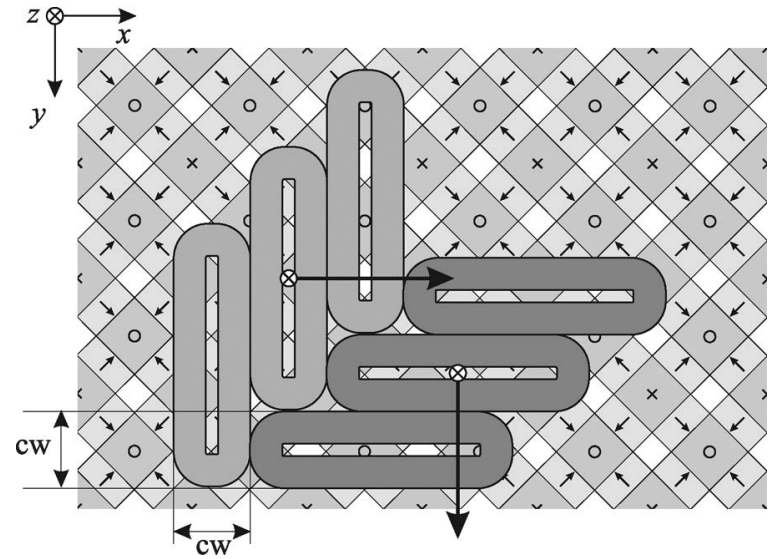

Fig. 6. Bottom view of a Halbach magnet array and two coil groups laid in a herringbone pattern $\left(c w=(4 / 3) \tau_{n}\right.$ and $\left.c l=4 \tau_{n}\right)$.

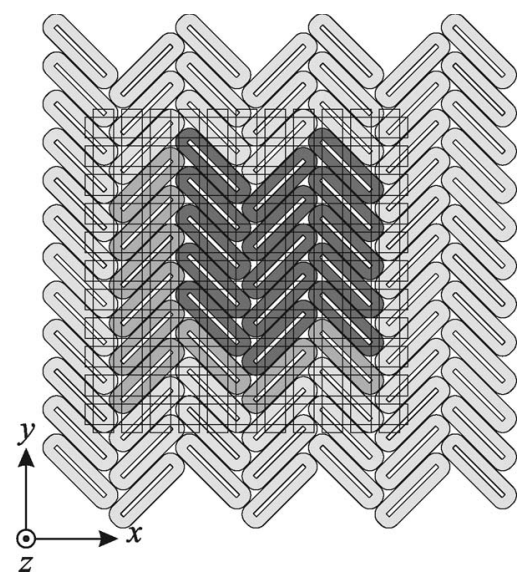

Fig. 7. Topology 1: 15-24 active coils, $c w=(4 / 3) \tau_{n}$, and $c l=4 \tau_{n}$.

\section{TOPOLOGY COMPARISON}

Four planar actuators have been synthesized and optimized. The translators are constructed of a carrier with a size of $300 \times$ $300 \mathrm{~mm}$, a mass of $4 \mathrm{~kg}$, and $\rho_{f}=1500 \mathrm{~kg} / \mathrm{m}^{3}$. The number of magnetic poles and, hence, the pole pitch depend on the number of active coils and the coil size. The sizes of the coils and the magnets in the Halbach magnet array in every topology are optimized with the described optimization procedure.

The coils in three planar actuators are laid in a herringbone pattern. A detail of a herringbone pattern is shown in Fig. 6. In the figure, a herringbone pattern with rectangular coils with $c w=(4 / 3) \tau_{n}$ is shown. Each coil is shifted over $c w$ in both the $x$ - and $y$-directions. The light and dark gray coil groups in Fig. 6 each form a three-phase system. The light gray system can produce a position-independent force vector in the $x z$ plane and the dark gray system in the $y z$ plane, as indicated in the figure. Coils can also be shifted over $c w=(3 / 2) \tau_{n}$ and form semi-four-phase systems. In that case, only two coils in a coil group are necessary to produce a position-independent force vector.

The three compared planar actuators with coils in a herringbone pattern are shown in Figs. 7-9. The coil length in topologies 1 and 2 is $c l=4 \tau_{n}$. The coils are distributed according to a three-phase $c w=(4 / 3) \tau_{n}$ and a semi-four-phase system

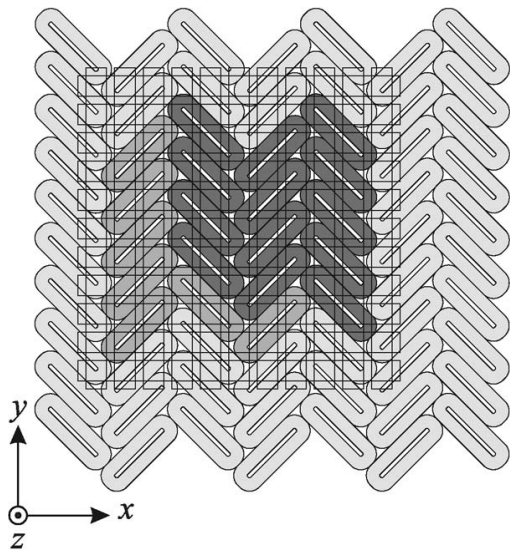

Fig. 8. Topology 2: 12-20 active coils, $c w=(3 / 2) \tau_{n}$, and $c l=4 \tau_{n}$.

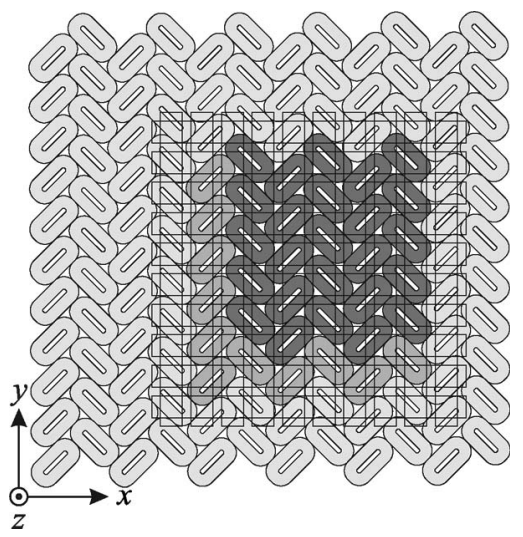

Fig. 9. Topology 3: 25-36 active coils, $c w=(4 / 3) \tau_{n}$, and $c l=2 \tau_{n}$.

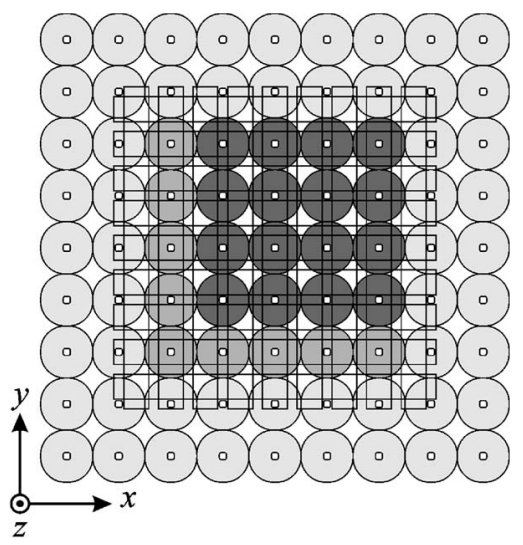

Fig. 10. Topology 4: 16-25 active coils and $c w=(3 / 2) \tau$.

$c w=(3 / 2) \tau_{n}$, respectively. The coils of topology $3\left(c l=2 \tau_{n}\right)$ are also distributed according to a three-phase system with $c w=(4 / 3) \tau_{n}$. Topologies 1 and 2 have a magnet array with $11 \times 11$ poles. Topology 3 requires only $10 \times 10$ poles. Therefore, the pole pitch of topology 3 is $10 \%$ larger. The fourth topology, shown in Fig. 10, is a planar actuator with square coils [3]. The coils are distributed according to a semi-four-phase system $(c w=(3 / 2) \tau)$. A group of $2 \times 2$ coils can produce a position-independent force vector in the $x y z$ plane.

In Figs. 7-10, the coils are indicated in three colors. At the position of the magnet array shown in the figures, both the dark gray and the black coils are active, and the light gray coils are 
inactive. The black coils show the minimal set of active coils when the translator is moved in the positive $x$ - and $y$-directions.

Simulation of a planar actuator within its full reachable volume is computationally demanding. To estimate the minimum and maximum power dissipation (ohmic losses) in the planar actuator, two different static load situations are analyzed: levitation of the translator only

$$
\vec{w}=[0(\mathrm{~N}), 0(\mathrm{~N}), m g(\mathrm{~N}), 0(\mathrm{Nm}), 0(\mathrm{Nm}), 0(\mathrm{Nm})]^{\top}
$$

and levitation and acceleration of the translator in the $x y$ plane with $10 \mathrm{~m} / \mathrm{s}^{2}$

$$
\vec{w}=[10 m(\mathrm{~N}), 10 m(\mathrm{~N}), m g(\mathrm{~N}), 0(\mathrm{Nm}), 0(\mathrm{Nm}), 0(\mathrm{Nm})]^{\top}
$$

where $m$ is the levitated mass. The force, torque, and ohmic losses in the coils have been predicted on a grid in the $x y$ plane, containing approximately 2000 points. The mechanical clearance between stator and translator (the air-gap) is equal to $1.0 \mathrm{~mm}$.

The force and torque are predicted with the harmonic model presented in [7]. In this model, the magnetization vector of the magnet array is described with a Fourier series that depends on two harmonic numbers $k$ and $l$ in orthogonal directions, where $k$ and $l$ are odd natural numbers. The end effects of the magnet array are not included in the model. The force and torque are calculated with the Lorentz force law. The volume integral over the coil is analytically solved in the straight segments of the coils and is reduced to a numerical surface integral in the corner segments. As a result, the force and torque in a planar actuator can be predicted in less than $1 \mathrm{~s}$ when up to the ninth harmonic is calculated. Because the weighing matrix $\Delta$ switches coils off before the force and torque are significantly influenced by the end effects of the magnet array, the use of the harmonic model, which assumes an infinitely large magnet array, does not result in significant errors in the predictions.

In Table II, the results of the comparison are summarized. The table shows that the topologies with a larger pole pitch (topologies 3 and 4 ) require higher magnets and, therefore, have a larger levitated mass.

The mean ohmic losses in the coils of planar actuator topology 4 with square coils are $28 \%$ larger than that in topology 1 with rectangular coils. The difference between the minimum and maximum power dissipation is the smallest for topology 3 with the most active coils. In this topology, 11 of the 36 coils $(31 \%)$ are switched off at the worst case transition between different coil sets.

The force and torque ripples in the planar actuators are small in the three topologies with rectangular coils (actuators 1,2, and 3). The rms force ripples in these topologies are less than $0.4 \%$ during levitation and acceleration. Topology 4 suffers from large force ripples up to $2.5 \%$. The force ripples are caused by higher harmonics in the magnetic flux density distribution. The magnitude of these harmonics can be influenced by changing the ratio of the sizes of the permanent magnets in the Halbach magnet array $\left(\tau_{m} / \tau\right)$. Using the harmonic model, the amplitude of the ripples caused by different higher harmonics (indicated by the harmonics numbers $k$ and $l$ ) is calculated
TABLE II

\begin{tabular}{|c|c|c|c|c|}
\hline Topology & 1 & 2 & 3 & 4 \\
\hline active coils (xy) & $4 \times 6$ & $4 \times 5$ & $6 \times 6$ & $5 \times 5$ \\
\hline magnetic poles (xy) & $11 \times 11$ & $11 \times 11$ & $10 \times 10$ & $9 \times 9$ \\
\hline$\tau(\mathrm{mm})$ & 25.0 & 25.0 & 27.4 & 30.4 \\
\hline$c w$ & $\frac{4}{3} \tau_{n}$ & $\frac{3}{2} \tau_{n}$ & $\frac{4}{3} \tau_{n}$ & $\frac{3}{2} \tau$ \\
\hline$c l$ & $4 \tau_{n}$ & $4 \tau_{n}$ & $2 \tau_{n}$ & $\mathrm{n} / \mathrm{a}$ \\
\hline \multicolumn{5}{|c|}{ Sizes obtained from optimization of single coil and magnet array } \\
\hline ch & $0.28 \tau$ & $0.28 \tau$ & $0.28 \tau$ & $0.28 \tau$ \\
\hline$c b$ & $0.56 \tau_{n}$ & $0.65 \tau_{n}$ & $0.59 \tau_{n}$ & $0.65 \tau$ \\
\hline & 0.66 & 0.66 & 0.66 & 0.66 \\
\hline$m h(\mathrm{~mm})$ & 7.1 & 7.1 & 7.6 & 8.0 \\
\hline mass $(\mathrm{kg})$ & 7.95 & 7.95 & 8.17 & 8.44 \\
\hline air-gap (mm) & 1.0 & 1.0 & 1.0 & 1.0 \\
\hline \multicolumn{5}{|c|}{ Levitation only: $\vec{F}=\left[\begin{array}{lll}0 & 0 & m g\end{array}\right]^{\top}(\mathrm{N}), \vec{T}=\overrightarrow{0}(\mathrm{Nm})$} \\
\hline Ohmic loss mean $(\mathrm{W})$ & $\overline{50.0}$ & 54.0 & 256.8 & 64.1 \\
\hline Ohmic loss min (W) & 41.4 & 40.1 & 49.3 & 44.2 \\
\hline Ohmic loss max (W) & 69.0 & 80.1 & 73.4 & 85.1 \\
\hline$F_{x}$ rms ripple $(\mathrm{N})$ & 0.049 & 0.057 & 0.116 & 0.757 \\
\hline$F_{y}$ rms ripple $(\mathrm{N})$ & 0.076 & 0.096 & 0.120 & 0.757 \\
\hline$F_{z}$ rms ripple $(\mathrm{N})$ & 0.080 & 0.079 & 0.182 & 2.143 \\
\hline$T_{x}$ rms ripple $(\mathrm{Nm})$ & 0.015 & 0.009 & 0.013 & 0.025 \\
\hline$T_{y}$ rms ripple $(\mathrm{Nm})$ & 0.014 & 0.015 & 0.010 & 0.025 \\
\hline$T_{z}$ rms ripple $(\mathrm{Nm})$ & 0.011 & 0.011 & 0.013 & 0.001 \\
\hline \multicolumn{5}{|c|}{ Acceleration and levitation: $\vec{F}=\left[\begin{array}{lll}10 m & 10 m & m g\end{array}\right]^{\top}(\mathrm{N}), \vec{T}=\overrightarrow{0}(\mathrm{Nm})$} \\
\hline Ohmic loss mean (W) & 278 & 302 & 303 & 359 \\
\hline Ohmic loss min (W) & 217 & 211 & 258 & 239 \\
\hline Ohmic loss max (W) & 588 & 627 & 443 & 635 \\
\hline$F_{x}$ rms ripple $(\mathrm{N})$ & 0.106 & 0.146 & 0.191 & 1.189 \\
\hline$F_{y}$ rms ripple $(\mathrm{N})$ & 0.218 & 0.298 & 0.183 & 1.189 \\
\hline$F_{z}$ rms ripple $(\mathrm{N})$ & 0.157 & 0.251 & 0.247 & 1.679 \\
\hline$T_{x}$ rms ripple $(\mathrm{Nm})$ & 0.029 & 0.027 & 0.022 & 0.061 \\
\hline$T_{y}$ rms ripple $(\mathrm{Nm})$ & 0.030 & 0.027 & 0.025 & 0.061 \\
\hline$T_{z}$ rms ripple $(\mathrm{Nm})$ & 0.028 & 0.026 & 0.024 & 0.013 \\
\hline
\end{tabular}

COMPARISON OF Four Planar ACTUATOR TOPOLOGIES

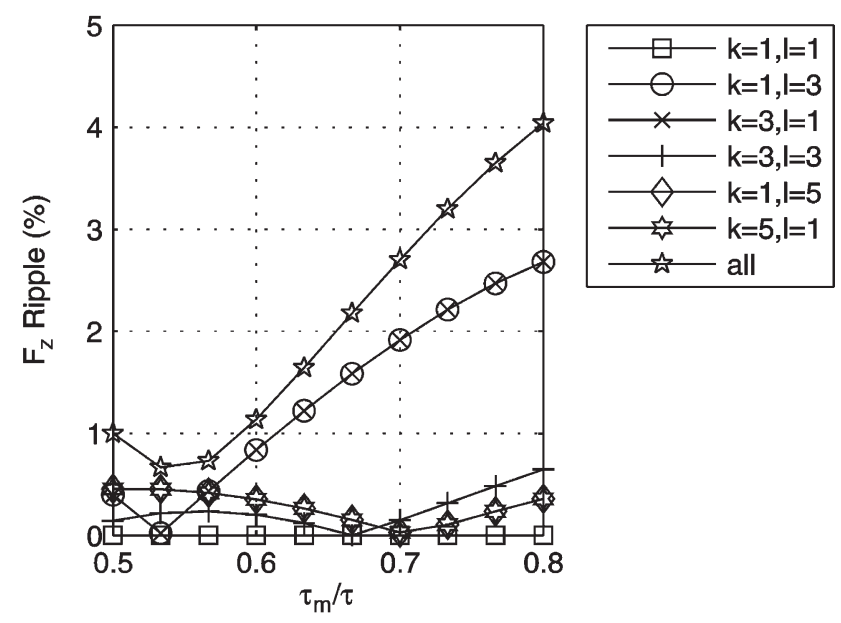

Fig. 11. Harmonic force ripple components of topology 4 versus $\left(\tau_{m} / \tau\right)$ $\left[\vec{F}=[10 m 10 m m g]^{\top}(\mathrm{N}), \vec{T}=\overrightarrow{0}(\mathrm{~N} \cdot \mathrm{m})\right]$

as a function of $\left(\tau_{m} / \tau\right)$. The calculations are carried out for the second static load. Although the amplitude of the ripples changes for different wrench vectors, the results, shown in Fig. 11, are typical for this actuator. The ripples in the actuator are mainly caused by the $k=1, l=3$ and the $k=3, l=1$ harmonics. Fig. 11 shows that the force ripples can significantly be reduced by choosing $\left(\tau_{m} / \tau\right)=0.53$. In that case, the $k=1$, $l=3$ and the $k=3, l=1$ harmonics do not occur in the spectrum of the magnetic flux density distribution. However, the force produced by the coil is also reduced, and the power 


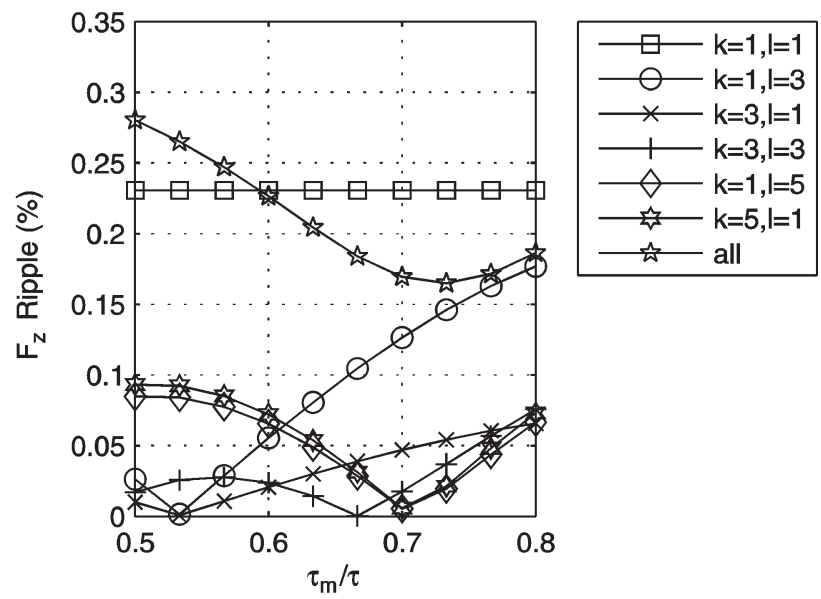

Fig. 12. Harmonic force ripple components of topology 1 versus $\left(\tau_{m} / \tau\right)$ $\left[\vec{F}=[10 m 10 m m g]^{\top}(\mathrm{N}), \vec{T}=\overrightarrow{0}(\mathrm{~N} \cdot \mathrm{m})\right]$.

TABLE III

Data of the Realized Planar Actuator

\begin{tabular}{|l|l|}
\hline \multicolumn{2}{|c|}{ Translator } \\
\hline Size $(x y)$ & $300 \times 300 \mathrm{~mm}$ \\
Pole pitch $(\tau)$ & $25 \mathrm{~mm}$ \\
Magnet height $(m h)$ & $7.0 \mathrm{~mm}$ \\
Ratio magnets Halbach array $\frac{\tau_{m}}{\tau}$ & 0.68 \\
Material magnets & VAC655HR \\
Mass & $8.2 \mathrm{~kg}$ \\
\hline \multicolumn{2}{|c|}{ Stator } \\
\hline Number of coils & 84 \\
Coil height $(c h)$ & $7.4 \mathrm{~mm}$ \\
Conductor bundle width $(c b)$ & $9.5 \mathrm{~mm}$ \\
Cooling medium & water \\
Power amplifiers & Prodrive (52V, 6A) \\
\hline \multicolumn{2}{|c|}{ Stroke } \\
\hline Stroke $(x y$-plane) & $230 \times 230 \mathrm{~mm}$ \\
Mechanical clearance & $1-2 \mathrm{~mm}$ \\
Rotation angles & $\pm 3 \mathrm{mrad}$ \\
Max. speed $(x y$-plane) & $1.4 \mathrm{~m} / \mathrm{s}$ \\
Max. acceleration $(x y$-plane) & $14 \mathrm{~m} / \mathrm{s}^{2}$ \\
\hline
\end{tabular}

dissipation in the actuator will rise with $9 \%$. Another way to reduce the force ripples is to include the $k=1, l=3$ and the $k=3, l=1$ harmonics in $\boldsymbol{\Gamma}(\vec{p})$. However, in that case the actuator will be more sensitive to commutation errors than when $\left(\tau_{m} / \tau\right)=0.53$.

The same type of analysis has been carried out for topology 1 with $45^{\circ}$ rotated magnets, which has a significantly lower force ripple in comparison with topology 4 . In this actuator, the ripple caused by the first harmonic of the magnetic flux density distribution ( $k=1, l=1)$ is dominant, as shown in Fig. 12. In most rotary and linear machines, a ripple caused by the first harmonic does not occur. However, in this actuator, it is assumed that the $x$ - and $y$-directions are fully decoupled in the mapping $\boldsymbol{\Gamma}(\vec{p})$. In reality, there is a crosstalk of approximately $0.8 \%$, which causes the force ripples. Changing the ratio $\left(\tau_{m} / \tau\right)$ does not influence the ripple caused by the first harmonic.

\section{STATUS}

Because of the low-power dissipation and small force and torque ripples, a planar actuator based on topology 1 has been manufactured. The dimensions and properties of the actuator are listed in Table III. Because of manufacturing reasons, the sizes are slightly different from the values in Table II.

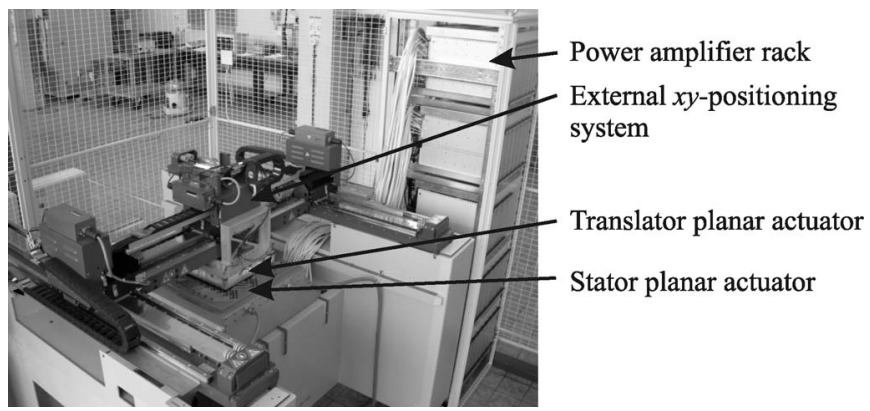

Fig. 13. Planar actuator and the external $x y$ positioning system.

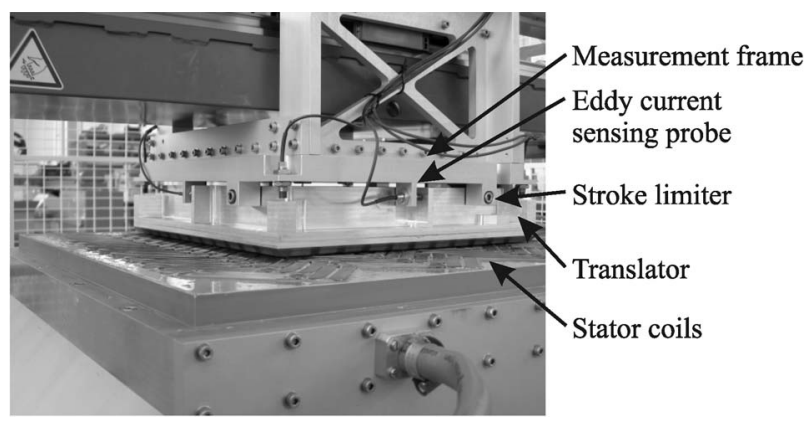

Fig. 14. Detail of the planar actuator and the measurement frame.

Fig. 13 shows an overview of the experiment. An external $x y$ positioning system (Assembléon H-drive) consisting of three linear motors moves along with the planar actuator. It is used for safety reasons and prevents damage to the actuator in case of unstable controllers or power failure. Fig. 14 shows a detail of the stator and translator of the planar actuator, and the measurement frame that is mounted to the $x y$ positioning system. The stroke of the translator with respect to the measurement frame is limited by four pins (diameter: $4.9 \mathrm{~mm}$ ) that fit in oversized holes in the translator (diameter: $6.0 \mathrm{~mm}$ ). During normal operation of the planar actuator, there is no contact between the translator and the measurement frame. The position of the translator is measured with eight eddy current sensing probes (LION ECL100-U8B) with respect to the $x y$ positioning system. The position and orientation of the translator of the planar actuator with respect to the coil array is reconstructed from the data of the eddy current sensing probes and the three encoders of the linear motors of the $x y$ positioning system.

The tracking error of the planar actuator has been determined with six single-input-single-output (SISO) quasiproportional-integral-differential (PID) controllers with mass and inertia feedforward and with a bandwidth of $35 \mathrm{~Hz}$. No multiple-input-multiple-output (MIMO) controller has been implemented because the wrench is sufficiently linearized and decoupled. When the translator is levitated $1.5 \mathrm{~mm}$ above the stator coils, the tracking error of the planar actuator is $30 \mu \mathrm{m}$ and $100 \mu \mathrm{rad}$ at full speed $(1.4 \mathrm{~m} / \mathrm{s})$ and full acceleration $\left(14 \mathrm{~m} / \mathrm{s}^{2}\right)$. At standstill operation, the rms tracking error is less than $0.1 \mu \mathrm{m}$ and $1 \mu \mathrm{rad}$.

\section{CONCLUSION}

A method for the design of a long-stroke moving-magnet planar actuator has been presented. Because the superposition 
principle can be applied to the force and torque production, the sizes of the magnets and coils in a planar actuator are determined by only optimizing the sizes of the magnet array and a single coil. Four planar actuators have been compared. The topologies with the $45^{\circ}$ rotated rectangular coils have a lower power dissipation and smaller force and torque ripples than the topology with square coils. The causes of the force ripples have been investigated by analyzing the harmonic spectrum of the forces. The topology with rectangular coils distributed according to a three-phase system has been manufactured. After linearization and decoupling, the actuator could accurately be controlled with six SISO controllers.

\section{ACKNOWLEDGMENT}

The authors would like to thank M. Uyt De Willigen for his assistance with the experimental work and ASML, Assembléon, Prodrive, Tecnotion, Vacuumschmelze, and the mechanical workshop of the Eindhoven University of Technology for their valuable contributions and support.

\section{REFERENCES}

[1] J. C. Compter, "Electro-dynamic planar motor," Precis. Eng., vol. 28, no. 2, pp. 171-180, Apr. 2004.

[2] W. J. Kim, D. L. Trumper, and J. H. Lang, "Modeling and vector control of a planar magnetic levitator," IEEE Trans. Ind. Appl., vol. 34, no. 6, pp. 1254-1262, Nov./Dec. 1998.

[3] A. J. Hazelton, M. B. Binnard, and J. M. Gery, "Electric motors and positioning devices having moving magnet arrays and six degrees of freedom," U.S. Patent 6208 045, Mar. 27, 2001.

[4] N. Korenaga, "Alignment apparatus and exposure apparatus using the same," U.S. Patent 7075 198, Jul. 11, 2006.

[5] J. C. Compter, P. C. M. Frissen, and J. van Eijk, "Displacement device," Patent WO 2006/075 291 A2, Jul. 20, 2006.

[6] C. M. M. van Lierop, J. W. Jansen, A. A. H. Damen, and P. P. J. van den Bosch, "Control of multi-degree-of-freedom planar actuators," in Proc. IEEE Int. CCA, Munich, Germany, Oct. 2006, pp. 25162521.

[7] J. W. Jansen, C. M. M. van Lierop, E. A. Lomonova, and A. J. A. Vandenput, "Modeling of magnetically levitated planar actuators with moving magnets," IEEE Trans. Magn., vol. 43, no. 1, pp. 15-25, Jan. 2007.

[8] C. R. Rao and S. K. Mitra, Generalized Inverse of Matrices and Its Applications. Hoboken, NJ: Wiley, 1971.

[9] J. P. Merlet, "Jacobian, manipulability, condition number, and accuracy of parallel robots,” J. Mech. Des., vol. 128, no. 1, pp. 199-206, 2006.

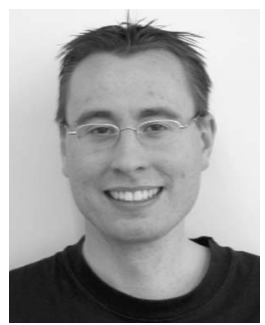

J. W. Jansen (S'03-M'07) received the M.Sc. degree (cum laude) in electrical engineering in 2003 and the Ph.D. degree in magnetically levitated planar actuator technology from Eindhoven University of Technology, Eindhoven, The Netherlands.

He then joined the Electromechanics and Power Electronics Group, Eindhoven University of Technology, where he is currently a Postdoctoral Researcher. His research interests include magnetic levitation and the analysis and design of linear and planar actuators.

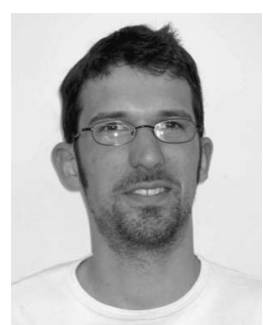

C. M. M. van Lierop (S'05-M'07) was born in Eindhoven, The Netherlands. He received the M.Sc. degree (cum laude) in electrical engineering in 2003 and the Ph.D. degree in magnetically levitated planar actuator technology from Eindhoven University of Technology, Eindhoven, The Netherlands.

He then joined the Control Systems Group, Eindhoven University of Technology, where he is currently a Postdoctoral Researcher. His main research interests deal with motion control, magnetic bearings, electron microscopy, and MIMO systems.

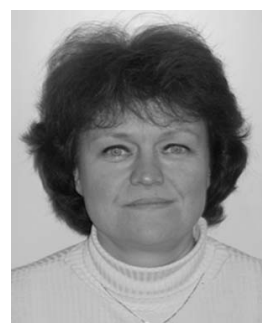

Elena A. Lomonova (M'04-SM'07) was born in Moscow, Russia. She received the M.Sc. (cum laude) and Ph.D. (cum laude) degrees in electromechanical engineering from Moscow State Aviation Institute (TU), Moscow, in 1982 and 1993, respectively.

In 2001, she joined Eindhoven University of Technology, Eindhoven, The Netherlands, as an Assistant Professor. She became an Associate Professor in 2008. She has worked on electromechanical actuator design, optimization, and development of advanced mechatronics systems.

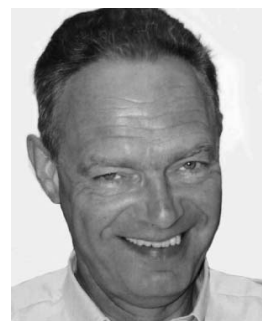

André J. A. Vandenput (M'77-SM'87) received the Ph.D. degree from the Catholic University of Leuven, Leuven, Belgium, in 1977.

From 1973 to 1991, he was with the Laboratory for Electrical Machines and Drives, Catholic University of Leuven, first as a Researcher of the Belgian National Fund for Scientific Research and, since 1987, as a Professor. From 1991 to 2008, he was a Full-Time Professor and Chairman of the section Electromechanics and Power Electronics at Eindhoven University of Technology, Eindhoven, The Netherlands. He obtained an Alexander von Humboldt-Stipendium for performing research at the RWTH Aachen, Aachen, Germany, from 1980 to 1981; was a Visiting Professor with the University of Colorado, Boulder, from 1983 to 1984; and was a Research Fellow with the Japan Society for the Promotion of Science (JSPS), University of Tokyo, Tokyo, Japan, in 1986. His teaching and research interests were high-performance electric drives, including electrical machines, power electronics, and control techniques. He passed away on January 11, 2008. 